\title{
The effectiveness evaluation of the carotenoids extraction from the tomatoes by means of absorption spectrophotometery method
}

\author{
Sergey Sokolov ${ }^{1}$, Innesa Deyneka ${ }^{2,}{ }^{*}$, Yuliya Katanaeva $^{1}$, Natia $_{\text {Ugrekhelidze }}{ }^{3}$, Elena \\ Yatskova $^{3}$, and Natalia Kulikova ${ }^{3}$ \\ ${ }^{1}$ Donetsk national University of Economics and trade named after Mikhail Tugan-Baranovsky, 31, \\ Shchorsa str., 83000, Donetsk, Ukraine \\ ${ }^{2}$ Lugansk National University named after Vladimir Dahl, 20a, Youth district, 91034, Lugansk, \\ Ukraine \\ ${ }^{3}$ Don State Technical University, 1, Gagarina sq, 344003, Rostov-on-Don, Russia
}

\begin{abstract}
In recent decades it is found that carotenoids have anticarcinogenic, cardioprotective, anti-atherogenic properties, and immunomodulating, radioprotective and anti-toxic effects. Discovered effect of carotenoids and lycopene, in particular, can be used to develop recommendations for prevention and treatment of those who are subjected to emergency situations of radiation and biological nature. This paper deals with spectrophotometery method for determining relative content of carotenoids in tomatoes of different ripeness levels. It is demonstrated that a wide complex absorption band within the wavelength ranging from 3500 to $6500 \AA$ is superposition of absorption bands for specific carotenoid pigments, which are a part of in vivo tomato pulp samples. According to additivity law of absorbency, the area under obtained curves represents value directly proportional to the thickness of layer under investigation and total concentration of carotenoids that allows to identify relative content of carotenoids in examined samples. It was concluded that developed method of samples preparing for measurements and the spectrophotometery method itself for determining the relative content of carotenoids in research tomatoes, as well as waste raw materials and oil cake, has shown a sufficient reliability, high speed and economic viability.
\end{abstract}

\section{Introduction}

Carotenoids, or carotenoid pigments, form one of the most numerous and very common groups of natural pigments that cause the color of many plants and animals. By their chemical nature, they rate as tetraterpenes class. Currently, it is known about 600 carotenoids, among which lycopene is especially noteworthy, which gives the red color to fruits of tomato, and it is commonly known that the color of the fruit is an important indicator of their quality and biological value.

\footnotetext{
*Corresponding author: Kaf_lipp@mail.ru
} 
In the human body carotenoids play an important role as initial products from which the vitamins of group A are formed. The authors [1] have reviewed the structural features of carotenoids, their nutritive sources, bioavailability and biotransformation in the body. Increasingly, carotenoids are used in medicine as physiologically active compounds, a source of vitamins, etc. In sufficient detail, data on the biological activity of the most common carotenoids are presented in considerable detail in reviews [2, 3, 4]. Upon that, the authors pay special attention to the anticarcinogenic, cardioprotective and antiatherogenic properties of carotenoids, as well as immunomodulating, radioprotective and antitoxic effects. In paper [5] the radioprotective effect of lycopene was studied. It turned out that lycopene protects cell membranes from the damaging effects of ionizing radiation. The discovered effect of carotenoids and, in particular, lycopene can be used to develop recommendations for the prevention and treatment of those who are subjected to the emergency situations of radiation and biological nature.

As a natural dye, beta-carotene, yellow-orange pigment of carrots, and its water-soluble derivatives are added to drinks, that makes them an additional source of vitamin A necessary for the organism. Other than beta-carotene, the red pigment of tomato lycopene is found in nature in very small quantities. Currently, the researchers, studying natural compounds, have enough modern analytical methods to specify the structure, to carry out identification, and to determine the quantitative content of carotenoids. Isolation of individual carotenoids from natural objects is directly related to the problem of specifying or confirming their structure. As a rule, the carotenoids structure determination is carried out by nuclear magnetic resonance spectroscopy [6,7]. This method is the most informative for the carotenoids structure specifying. Quite often, the infrared spectrometry method is used for their identification, allowing to confirm structural fragments and functional groups $[8,9]$. For the analysis of carotenoids, methods of near infrared spectroscopy, Raman spectroscopy and mass spectroscopy are used in combination with high performance liquid chromatography [10-12]. However, all these methods are time consuming and expensive.

The purpose of the work is to develop and justify an inexpensive, informative and fast, spectrophotometric method for determining the relative content of carotenoids in tomato fruits with various degrees of maturity.

\section{Materials and methods}

The following equipment and software were used to implement this method: the improved PGS-2 single-beam spectrograph (Carl Zeiss); 170W tungsten filament lamp with a color temperature of $1630 \mathrm{~K}$; a photodetector on the basis of a spectrophotometric detector SPhD-1 with a photodiode FDUK-100UT [13] and an automated system for data documenting and analyzing.

The relative content of carotenoids was determined by absorption spectrophotometry in the wavelength range from 3500 to $10000 \AA$. The investigation is concerned within in vivo tomato fruits pulp, dried pulp of tomato fruit and tomato paste. Samples for measurements were prepared in accordance with the procedure described below:

1. It is selected 10 fruits if this is a case of tomato fruits pulp in vivo which parameters and properties fully meet the relevant requirements of this tomato variety.

2. It is taken $1 \mathrm{~cm}^{3}$ of pulp without peel and seeds from each fruit, they are thoroughly ground and mixed together until a homogeneous pasty mixture is formed.

3. It is formed a layer of the obtained mixture $0.6 \mathrm{~mm}$ thick between plane-parallel colorless and transparent quartz plates.

4. It is formed a layer with a thickness of $0.1 \mathrm{~mm}$ if this is a case of dried tomato pulp prepared as a polydisperse powder with an equivalent particle diameter no more than 0.05 $\mathrm{mm}$. 
5. The layer thickness is equal to $0.6 \mathrm{~mm}$ if this is a case of tomato paste.

6. Quartz plates with the prepared sample are installed in the optical cuvette of the improved single-beam spectrograph PGS-2 and transmission spectra are measured.

\section{Results}

The samples prepared for measurements are polydisperse multiphase light-scattering and light-absorbing systems. Intensity attenuation of the light flux during transmitting through such a system occurs as a result of the interaction of electromagnetic radiation with absorbing and scattering centers in the system and depends on their quantity. Whenever the sample is a plane-parallel and homogeneous substance layer, then part of the light flux is also reflected from the interface. Fig. 1 schematically shows the processes of electromagnetic radiation interaction when transmitting through a plane-parallel and homogeneous layer of light-scattering and light-absorbing substances.

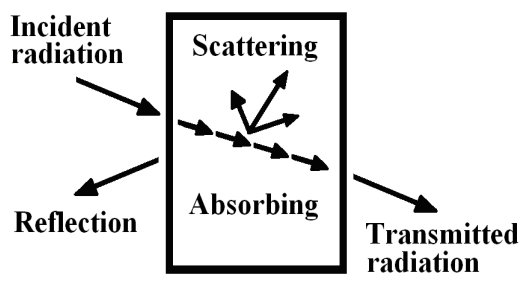

Fig. 1. Scheme of the interaction processes for electromagnetic radiation when transmitting through a plane-parallel and homogeneous layer of light-scattering and light-absorbing substance.

The intensity of the light flux, transmitted through a plane-parallel and homogeneous layer of light-scattering and light-absorbing substance, we will present as:

$$
I_{\text {trans. }}=I_{\text {incrad }}-\left(I_{\text {abs. }}+I_{\text {refl }}+I_{\text {scat }}\right)
$$

where $I_{\text {inc rad }}$-radiation rate, incident on substance layer; $I_{\text {abs. }}-$ the intensity of the radiation absorbed by the substance; $I_{\text {refl. }}$ - the intensity of the radiation reflected from the interface; $I_{\text {scat. }}$ - the intensity of radiation scattered by the substance in any directions; $I_{\text {trans. }}$ - the intensity of the radiation, transmitted through the layer of substance.

In comparative measurements of the absorbed radiation energy by different samples, identical plane-parallel colorless and transparent quartz plates were used, for which the intensity of radiation reflected from the interface is constant, has a relatively small value, and therefore is not taken into account (Iot $\approx 0$ ). Then equation $(1)$ will be as:

$$
I_{\text {trans. }}=I_{\text {incrad }}-\left(I_{\text {abs. }}+I_{\text {scat }}\right)
$$

Thus, luminous flux declension when transmitting through a plane-parallel and homogeneous layer of polydisperse multiphase light-scattering and light-absorbing samples is caused by scattering and absorption. Herewith it is accepted that the absorption and scattering of light are independent processes of the radiation interaction with particles of the dispersed phase and considered the scattered radiation to be fictitiously absorbed. At that the basic law of light declension of the Bouguer-Lambert-Behr which expresses therelationship between the intensities of the incident and transmitted electromagnetic radiation flows will have the following form:

$$
I_{\text {Trans. }}=I_{\text {incrad }} \cdot \exp [-(\varepsilon(\lambda)+k(r)) \cdot C \cdot d]
$$


where $\varepsilon(\lambda)$ is the molar coefficient of light absorption, depending on temperature, pressure and wavelength $\lambda$ of electromagnetic radiation; $k(r)$ is the coefficient of fictitious absorption (light flux intensity declension) caused by light scattering of dispersed particles with a radius $r ; C$ is the molar concentration of the light-absorbing phase, [mol/l]; $\mathrm{d}$ is the thickness of the layer [cm].

Taking the logarithm and transforming the law (3), we obtain the expression for the natural optical density:

$$
\mathrm{D}=\ln \frac{I_{\text {incrad }}}{I_{\text {Trans. }}}=(\varepsilon(\lambda)+k(r)) \cdot C \cdot d=\mathrm{D}_{\varepsilon}+\mathrm{D}_{k}
$$

where $\mathrm{D}_{\varepsilon}$ - optical density, underpinned by absorption, $\mathrm{D}_{k}-$ optical density, underpinned by light scattering.

From expression (4) it follows, firstly, that the relationship between the optical density $\mathrm{D}$, concentration $C$ and the layer thickness $d$ is directly proportional, and secondly, that the optical density $\mathrm{D}$ is independent of the light flux energy $I$. The light-absorbing and lightscattering properties of the substance characterize, respectively, the coefficients $\varepsilon(\lambda)$ and $k(r)$, which, like $\mathrm{D}$, are independent of the light intensity.

In the case when the composition of a polydisperse multiphase light-absorbing and light-scattering substance includes $\mathrm{N}$ of non-interacting dispersed phases, then the additivity law is observed for optical density:

$$
\mathrm{D}_{\text {com. }}=\sum_{i=1}^{\mathrm{N}} \mathrm{D}_{i}=d \cdot \sum_{i=1}^{\mathrm{N}}\left[\left(\varepsilon_{i}(\lambda)+k_{i}\left(r_{i}\right)\right) \cdot C_{i}\right]=\sum_{i=1}^{\mathrm{N}}\left[\mathrm{D}_{\varepsilon_{i}}+\mathrm{D}_{k_{i}}\right]
$$

where $\mathrm{D}_{\varepsilon i}$ - the optical density of the $i$-dispersed phase underpinned by absorption, $\mathrm{D}_{k i}$ - the optical density of the $i$-dispersed phase underpinned by light scattering.

The experimental assembly for recording the light source radiation spectrum and transmission spectra of samples at a constant room temperature of $295 \mathrm{~K}$ and atmospheric pressure is shown in Fig. 2.

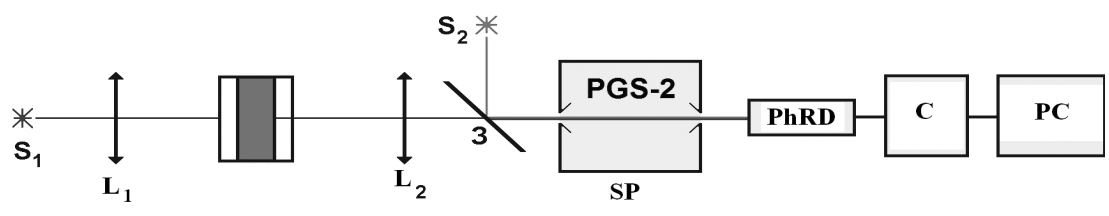

Fig. 2. Scheme of an experimental assembly for recording the light source radiation spectrum and transmission spectra of samples of a tomato fruit dried pulp, tomato fruits pulp of various maturity and tomato paste at a constant room temperature of $295 \mathrm{~K}$ and atmospheric pressure.

A source of continuous naturally polarized electromagnetic radiation is a $170 \mathrm{~W}$ tungsten filament lamp with a color temperature of $1630 \mathrm{~K}\left(\mathrm{~S}_{1}\right)$. Upon registration the radiation spectrum of the source, the light from the lamp $S_{1}$ transmits through the cuvette without a sample and is focused by a system of quartz capacitors $\left(\mathrm{L}_{1}\right.$ and $\left.\mathrm{L}_{2}\right)$ in the plane of the entrance slit of the PGS-2 spectrograph (SP). The SP spectrum, obtained in the plane of the exit slit, is recorded by a photo-receiving device based on the SFD-1 spectrophotometric detector [14]. Upon registration the transmission spectra, the radiation from the lamp $S_{1}$ transmits through a cuvette with the test sample (Sample). The output signal from SFD-1 in digital form enters on the controller input (C), which provides digital data processing and transmission of measurement results to a personal computer (PC). 
Recording and processing of data, coming from controller, as well as a graphical display of the measurement results of the FPU based on SFD-1, is carried out using software. The emission band of the green laser LED $\left(\mathrm{S}_{2}\right)$ with a maximum at $\approx 5319.48 \AA$, applied to the spectrograph input slit using a mirror $(\mathrm{M})$, is used as a ranging mark to calibrate the measured spectra according to wavelengths.

The recorded emission spectrum of a $170 \mathrm{~W}$ tungsten filament lamp with a color temperature of $1630 \mathrm{~K}$ and the transmission spectra of samples of tomato fruit dried pulp, tomato fruits pulp of various maturity and tomato paste are presented in Fig. 3.

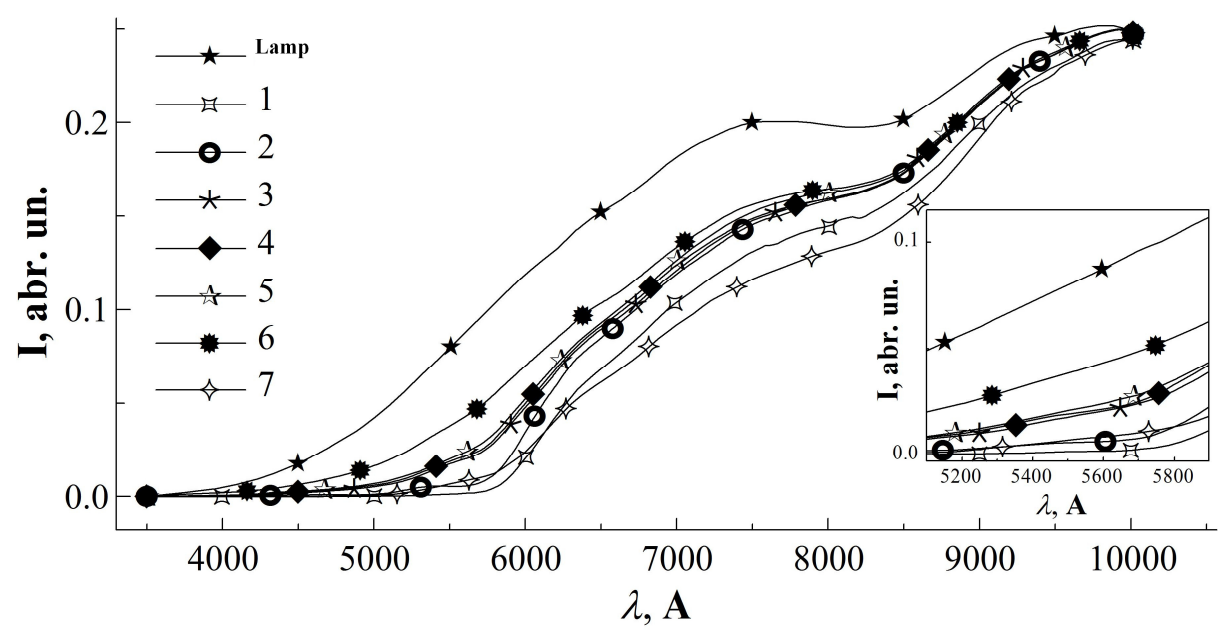

Fig. 3. The emission spectrum of a $170 \mathrm{~W}$ tungsten filament lamp with a color temperature of $1630 \mathrm{~K}$ (Lamp) and transmission spectra of samples: tomato fruit dried pulp (1), tomato fruits pulp of various maturity in descending order (2-6), tomato paste (7). In the figure the intensity of the emission spectrum of a tungsten filament lamp is reduced by 17 times.

The spectral dependences of the natural optical density D of the samples were calculated according to the measured transmission spectra using expression (4). The calculation result is shown in Fig. 4.

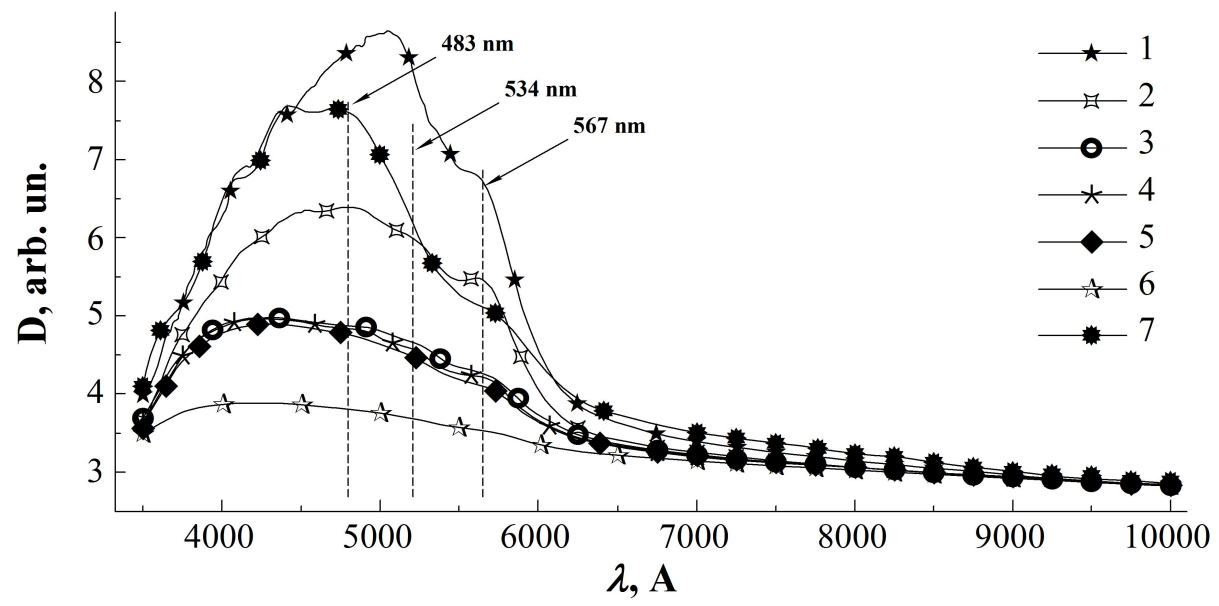

Fig. 4. Spectral dependences of the natural optical density D of the samples: tomato fruit dried pulp (1), pulp of tomato fruit of various maturity in descending order (2-6), tomato paste (7).

As can be seen in Fig. 4, in the wavelength range from 3500 to $6500 \AA$, a wide complex absorption band is observed, subsequent upon the absorption bands superposition of 
individual carotenoid pigments that are part of in vivo samples of tomato fruits pulp. The figure also shows a pronounced dependence of the absorption band intensity on the degree of tomato fruits maturity, which is associated with a change in the quantity of carotenoids in tomato fruits during their maturation. Thus, the highest intensity of the absorption band corresponds to the sample of pulp of the ripest tomato fruit (curve 2 in Fig. 4), which contains the most of carotenoids. Together with an increase in the intensity of the absorption band during the ripening of tomato fruits, its fine structure, which involves the occurring of three peaks with maxima at 483, 524 and $567 \mathrm{~nm}$, becomes more visible. With a high degree of probability, this can be associated with an expansion of contribution to the general curve D $(\lambda)$ of the carotenoid pigment lycopene, which has pronounced three component absorption spectra of solutions [15].

The experimental curves in Fig. 4 do not contain any pronounced absorption bands in the wavelength range from 6500 to $7500 \mathrm{~A}$, where long-wavelength absorption bands for chlorophylls are observed. This suggests an almost complete lack of chlorophyll in the in vivo samples of pulp tomato fruits [16-17].

As in vivo samples of the tomato fruits pulp are polydisperse multiphase systems consisting of light scattering and absorbing centers of irregular shape and various sizes, the strict consideration of light scattering processes is an extremely difficult task. However, if we take into account that law (3) is applicable to systems with a colloidal degree of dispersion at low concentrations and small layer thicknesses, that is wholly compliant with test samples of tomato fruit pulp in vivo, then at its simplest the spectral dependence of optical density $D_{k}(\lambda)$, caused by light scattering, is allowed to approximate by empirical expression in the form of a linear function:

$$
\mathrm{D}_{k}(\lambda)=\mathrm{a}+\mathrm{b} \cdot \lambda
$$

where $a$ and $b$ - coefficients independent of the wavelength $\lambda$ [18-19].

The numerical values of the coefficients of expression (6) calculated for the samples by the least square method are shown in Table 1.

Table 1. The values of the coefficients of expression (6) for dried tomato fruit pulp, tomato fruits pulp of various maturity and tomato paste samples (Fig. 4).

\begin{tabular}{|c|c|c|}
\hline \multirow{2}{*}{ Sample } & \multicolumn{2}{|c|}{ Coefficient } \\
\cline { 2 - 3 } & $\mathbf{a}$ & $\mathbf{b}$ \\
\hline 1 & 4.65020 & -0.000190391 \\
\hline 2 & 4.10343 & -0.000132442 \\
\hline 3 & 4.14771 & -0.000138624 \\
\hline 4 & 4.04764 & -0.000126603 \\
\hline 5 & 3.96568 & -0.000117669 \\
\hline 6 & 3.85627 & -0.000104717 \\
\hline 7 & 4.78853 & -0.000199296 \\
\hline
\end{tabular}

Using the obtained coefficients (Table 1), the intensity of the scattered radiation was determined and the spectral dependences of the optical density D of the samples were calculated taking into account the light scattering. The calculation result is shown in Fig. 5.

The experimental dependences in Fig. 5 make it possible to determine the relative content of carotenoids in examined samples. Since the wide complex absorption band in the wavelength range from 3500 to $6500 \mathrm{~A}$ is a superposition of the absorption bands of individual carotenoid pigments that are part of in vivo samples of tomato fruit pulp, then in accordance with the law of optical density additivity D (5), the area under the curves is a 
value directly proportional to the thickness of layer $d$ and the total concentration of carotenoids $C_{\text {amounts }}=\sum_{i=1}^{\mathrm{N}} \tilde{N}_{i}$.

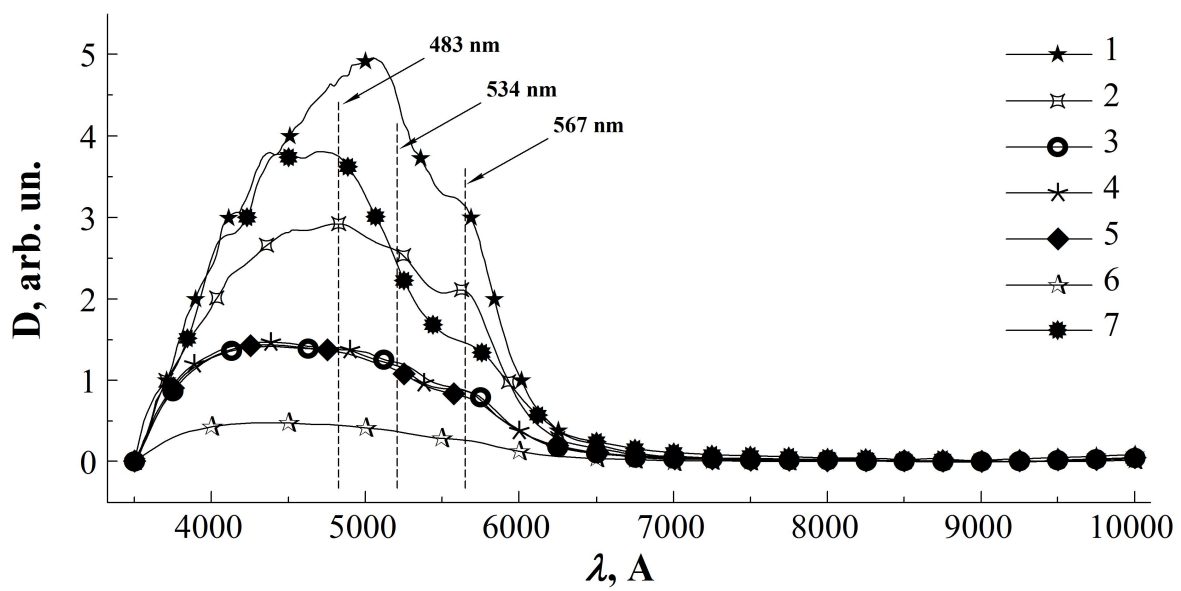

Fig. 5. Spectral dependences of the natural optical density D for samples of tomato fruit dried pulp (1), tomato fruits pulp of various maturity in descending order (2-6) and tomato paste (7) taking into account the light scattering.

The results of the calculation for the relative total concentration of carotenoids $C_{\text {amounts }}^{\text {relative }}=\frac{\sum_{i=1}^{\mathrm{N}} \tilde{N}_{i}}{C_{\text {amounts }}^{\max }}$ in the samples of tomato fruit dried pulp (1), tomato fruits pulp of various maturity (2-6) and tomato paste (7), are summarized in the table 2.

Table 2. Calculation data for relative total concentration of carotenoids $C_{\text {amounts }}^{\text {relative }}$ in the samples of tomato fruit dried pulp (1), tomato fruits pulp of various maturity (2-6) and tomato paste (7).

\begin{tabular}{|c|c|c|}
\hline Sample & $C_{\text {amounts }}^{\text {relative }}$ & $C_{\text {amounts }}^{\text {relative }} \cdot 100, \mathbf{\%}$ \\
\hline 1 & 1.00000 & 100 \\
\hline 2 & 0.11150 & 11.150 \\
\hline 3 & 0.05876 & 5.876 \\
\hline 4 & 0.05876 & 5.876 \\
\hline 5 & 0.05724 & 5.724 \\
\hline 6 & 0.01909 & 1.909 \\
\hline 7 & 0.12686 & 12.686 \\
\hline
\end{tabular}

\section{Conclusion}

The developed method of samples preparing for measurements, and the spectrophotometric method itself for determining the relative content of carotenoids in tomato fruits, as well as in waste materials and oilcake, showed sufficient reliability, high speed of research and economic viability. 


\section{References}

1. V.A. Dadali, V.A. Tutelyan, Yu.V. Dadali, L.V. Kravchenko, Nutrition Issues 79(2), 418 (2010)

2. V.A. Dadali, Yu.V. Dadali, V.A. Tutelian, L.V. Kravchenko, Nutrition issues 80(4), 418 (2011)

3. K. Sahana, Research Journal of Pharmacy and Technology 8(8), 1043-1047 (2015)

4. M.Ya. Shashkina, P.N. Shashkin, A.V. Sergeev, Russian Biotherapeutic Journal 9(1), 77-86 (2010)

5. F. Boehm, R. Edge, T. George Truscott, C. Witt, Febs latters 590(8), 1039-1288 (2016)

6. C.R. Gale, H.E. Ashurst, H.J. Powers, C.N. Martyn, American Journal of Clinical Nutrition 74(3), 402-408 (2001) PMid:11522566

7. R. Silverstein, F. Webeter, D. Kim, Spectrometric identification of organic compounds / (BINOM. Laboratory of Knowledge, 2011)

8. S. Sokolov, Y. Katanaeva, Production and Processing of Agricultural Products: Quality and Safety Management. Materials of an international scientific-practical conference dedicated to the 25th anniversary of Technology and Commodity Research Faculty of Voronezh State Agrarian University named after Emperor Peter I, 71-77 (2018)

9. V.F. Drobotko, V.N. Varyukhin, G.V. Bukin, S.A. Sokolov, V.P. Golovinov, Physics and technology of high pressures 28(4), 22-38 (2018)

10. A.G. Kuregyan, Basic research 2(23), 5166-5172 (2015)

11. L.A. Nafie, Journal of Raman Spectroscopy 48(12), 1692-1717 (2017)

12. J. Qin, M.S. Kim, K. Chao, S. Dhakal, B.-K. Cho, S. Lohumi, C. Mo, Y. Peng, M. Huang, Postharvest Biology and Technology 149, 101-117 (2019) DOI: 10.1016/j.postharvbio.2018.11.004

13. V. Sukhanov, P. Aruev, M. Drozdova, N. Zabrodskaya et al., FTP 47(2), 174 (2013)

14. V. Sukhanov, V. Zabrodsky, P. Aruev, E. Sherstnev, P. Vtulkin, S. Marchenko, Photonics 1, 75-84 (2014)

15. S.A. Sokolov, N.N. Sevatorov, Yu.A. Katanaeva, V.V. Zabrodsky, V.F. Drobotko, Equipment and technologies for food production: thematic collection of scientific works, Donetsk 1(34), 30-37 (2017)

16. S.I. Kambulov, I.V. Bozhko, A.V. Olshevskaya, MATEC Web of Conferences 224, 05022 (2018) https://doi.org/10.1051/matecconf/201822405022

17. A. Altybayev, A. Zhanbyrbayev, B. Meskhi, D. Rudoy, A. Olshevskaya, A. Prohorova, E3S Web of Conferences 135, 01078 (2019) https://doi.org/10.1051/e3sconf/201913501078

18. G. Parkhomenko, S. Kambulov, A. Olshevskaya, A. Babadzhanyan, N. Gucheva, I. Mekhantseva, IOP Conf. Series: Earth and Environmental Science 403, 012144 (2019) doi:10.1088/1755-1315/403/1/012144

19. Y. Lachuga, A. Soloviev, A. Matrosov, I. Panfilov, V. Pakhomov, D. Rudoy, IOP Conf. Series: Earth and Environmental Science 403, 012055 (2019) doi:10.1088/1755$1315 / 403 / 1 / 012055$ 